\title{
EFFECTS OF DEPRESSIVE DISORDER ON FALSE MEMORY FOR EMOTIONAL INFORMATION
}

\author{
Zai-Ting Yeh, Ph.D., ${ }^{1}$ and Mau-Sun Hua, Ph.D. ${ }^{2 *}$
}

\begin{abstract}
Background: This study explored with a false memory paradigm whether (1) depressed patients revealed more false memories and (2) whether more negative false than positive false recognition existed in subjects with depressive disorders. Methods: Thirty-two patients suffering from a major depressive episode (DSM-IV criteria), and 30 age-and education-matched normal control subjects participated in this study. After the presentation of a list of positive, negative, and neutral association items in the learning phase, subjects were asked to give a yes/no response in the recognition phase. They were also asked to rate 81 recognition items with emotional valence scores. Results: The results revealed more negative false memories in the clinical depression group than in the normal control group; however, we did not find more negative false memories than positive ones in patients. When compared with the normal group, a more conservative response criterion for positive items was evident in patient groups. It was also found that when compared with the normal group, the subjects in the depression group perceived the positive items as less positive. Conclusions: On the basis of present results, it is suggested that depressed subjects judged the emotional information with criteria different from normal individuals, and patients' emotional memory intensity is attenuated by their mood. Depression and Anxiety 26:456-463, 2009. (C) 2009 Wiley-Liss, Inc.
\end{abstract}

Key words: depressive disorder; false memory; mood-congruence memory bias; emotional information

\section{INTRODUCTION}

$\mathbf{I}_{\mathrm{n}}$ the study of the relationship between emotion and memory, past research ${ }^{[1-4]}$ has shown that depressed people have a memory bias for emotional information. The main theoretical framework regarding such a bias is mood-congruent memory $(\mathrm{MCM})^{[5-7]}$, which is defined as the tendency to recall information that is conceptually congruent with one's mood. According to this model, subjects with depression will exhibit a pronounced bias and thus recognize negative valence materials more efficiently than positive or neutral ones.

The MCM bias provides a cognitive framework for explaining the emergence and maintenance of depression. ${ }^{[8]}$ An interesting question is whether depressed people also show an increase in the number of moodcongruent false recognitions; that is, a patient may report that he or she remembers something that did not actually happen. Previous studies used veridical memory measurements to explore MCM bias. This study suggests that the way that memory impacts cognitive function may not always be truthful. Many of

\footnotetext{
1Department of Clinical Psychology, Fu Jen Catholic University, Taipei, Taiwan

2Department of Psychology, National Taiwan University, Taipei, Taiwan

Contract grant sponsor: National Science Council, Taipei, Taiwan; Contract grant number: NSC92-2413-H-002-011; NSC93-2413$\mathrm{H}-002-013$; Contract grant sponsor: Cognitive Neuroscience Development Grant.

*Correspondence to: Mau-Sun Hua, Ph.D., Department of Psychology, National Taiwan University, Taipei 106, Taiwan. E-mail: huams@ntu.edu.tw

Received for publication 28 August 2007; Revised 31 October 2007; Accepted 1 November 2007

DOI 10.1002/da.20453

Published online 25 February 2009 in Wiley InterScience (www. interscience.wiley.com).
} 
the well-documented negative thinking biases in affective disorders are supported through cognitive distortions. Examples of such mechanisms are overgeneralization ${ }^{[9,10]}$ and rumination. ${ }^{[1-14]}$ Overgeneralization could be seen as the result of a depressed person's unjustified generalizations on the basis of a single incident leading to inaccurate conclusions when they retrieve memories. ${ }^{[15]}$ It has been found that overgeneralization of negative events predicts more subsequent symptoms of depressed individuals ${ }^{[9,10]}$ The second phenomenon, rumination, is defined as "repetitive behaviors and thoughts that focus one's attention on one's depressive symptoms and on the implications of these symptoms".[16] Studies with depressed patients suggest that rumination reduces the specificity of memories ${ }^{[17]}$ and thus enhances the probability of false memories. The cognitive styles of overgeneralization and rumination could make untruthful memories more accessible for recollection; thus, more false recognition would be expected.

There has been a great deal of interest in false memories because false memory studies provide a research mechanism by which one can distinguish interpretive memories and what actually happened in the real world. ${ }^{[18]}$ Studies on some clinical cases, such as schizophrenia, ${ }^{[19]}$ amnesia, ${ }^{[20]}$ Alzheimer dementia, ${ }^{[21,22]}$ as well as normal adults ${ }^{[23]}$ and aging ${ }^{[24]}$ have used the false memory paradigm to investigate memory system function. The most commonly used false recognition task is the Deese-Roediger McDermott (DRM) paradigm. ${ }^{[25,26]}$ In the typical procedure, lists of semantic related items are presented to subjects (for example: bed, rest, awake, tired, dream, wake, night, blanket, and doze). After the presentation of a series of such lists, they are asked to give a yes/no response in the later recognition test, which includes one nonpresented but semantically converging of the so-called critical lure or false target (for example, Sleep). ${ }^{[26]}$ found that healthy subjects had a high proportion of "yes" responses to false targets in a later recognition trial.

In this study, we employed a total of 27 lists including positive, negative, and neutral emotional valences (all were matched with word frequency and association strength) to explore false memories in patients with depression disorders. Subjects were instructed to memorize lists of words in the learning phase and in the later recognition phase they were asked to judge whether they saw the items before. False memory was scored on whether subjects said "yes" to items that did not appear in the learning phase. Furthermore, in accordance with the signal detection theory of recognition memory measure- ment, ${ }^{[27]}$ we also investigated the differences between depression and normal control groups in the memory discrimination ability (the ability to judge the items were new or old ones in the recognition phase) and response criterion index.

In summary, based on the cognitive distortion of depression, it was predicted that more false memories would be recorded in patients with depression disorders than in a normal control group. The second aim was to examine whether the depressed patients indeed activate negative association memories, as MCM suggests. If this is the case, it was expected that the negative false memories outnumber the positive ones in patients with depression. Third, we also hypothesized that memory discrimination is impaired for negative than for positive items in the depression groups. And fourth, a more liberal response criterion for negative items than for positive ones in the depression groups was expected in this study.

\section{METHODS}

\section{PARTICIPANTS}

Two groups of subjects were recruited in this study: clinically depressed patients $(N=32)$ and nondepressed controls $(N=30)$. All depressed outpatients were diagnosed according to the DSM-IV criteria by trained psychiatrists using the MINI psychopathological interview. ${ }^{[28]}$ The patients did not have any psychotic, neurological disorders, manic or hypomanic episodes, or substance abuse problems. The Beck Depression Inventory (BDI) was administered after the experiment. There were eight males and 24 females $(B D I=24.7 \pm 4.6)$ with a mean age of 31.6 years and a mean of 13.9 years of education.

Nondepressed controls $(\mathrm{BDI}=6.8 \pm 3.9)$ were recruited from among employees of the Mackay Memorial Hospital and matched for sex (eight males and 22 females), age (mean $=32.1$ ), and education $($ mean $=14.5)$. Healthy participants were also screened for psychopathological disturbances or substance abuse using the MINI interview, ${ }^{[28]}$ BDI assessment was also carried out after the experiment. They were all free of any medication and had no history of drug abuse, psychiatric illness, neurological disease, or major physical illness. Table 1 shows the demographic and clinical data of depression and normal healthy control groups.

Ethical approval for the study was given by the Research Ethics Committee at the Mackay Memorial Hospital, and all subjects signed informed consent forms before their participation.

\section{MATERIALS}

\section{STIMULUS WORDS}

The free association norm of 1,200 Chinese words from ${ }^{[29]}$ was used to construct 27 lists of semantically related items. Each list consisted of 13 association items (such as fulfillment, happiness, family,

TABLE 1. Demographic and clinical data of the depression and control groups

\begin{tabular}{lccccc}
\hline & Age (years) & Gender $(\mathrm{M}: \mathrm{F})$ & Education (years) & BDI & Illness duration (years) \\
\hline Depression $(N=32)$, Mean $(S D)$ & $31.6(6.5)$ & $8: 24$ & $13.9(2.8)$ & $24.7(4.6)$ & $9.8(3.2)$ \\
Control $(N=30)$, Mean $(S D)$ & $32.1(7.2)$ & $8: 22$ & $14.5(3.5)$ & $6.8(3.9)$ & - \\
\hline
\end{tabular}

M, male; F, female; BDI, Beck Depression Intervention. 
marriage, value, life, smile, couple, warmth, love, heart, and riches), which converged into one critical lure or false target word (such as welfare). The data of the critical lures' emotional value scores also came from the free association norm of. ${ }^{[29]}$ Scores of +3 to +1 represent very positive to slightly positive emotional values, a score of 0 represents no emotional value, and scores of -3 to -1 represent very negative to slightly negative emotional values, respectively. The 27 critical lures consisted of nine positive words with emotional values above +1.5 (art, reward, satiation, passion, respect, braveness, welfare, romantic, and tenderness), nine negative words with emotional values below -1.5 (typhoon, censure, sufferance, violence, pollution, annoyance, suicide, destruction, and deterioration), and nine neutral words (glasses, tourist, spread, road, category, business owner, revolve, cost, railway) with emotional values near zero.

All of the 351 items were two-character phrases written in Chinese. There were no significant differences between any two lists in the frequency of occurrence in common Chinese usage $[F(2,24)=0.21$, $P=.816] .^{[30]}$

The association strength between critical lures to items (forward association strength): the free association frequency from the free association norm was the index of association strength. For example, when presented with 'romantic', as a critical lure, 25 of 200 people would associate it with 'lover' (positive list); when presented 'typhoon', 26 of 200 people would associate it with 'disaster' (negative list). Those 12 association items were ordered by association strength to critical lure. Romantic (the critical lure) had the stronger association to lover (25) than candle (20), rose (12), and so on. The means of association strength are a total sum of the association strength of those 12 items divided by 12, which was calculated for each list separately. Nine positive lists (mean of association strength $=8.05$ ), nine negative lists (mean of association frequency $=7.79$ ), and nine neutral lists (mean of association frequency $=9.11)$ were chosen according to their equalization of the association strength of each list $[F(2,24)=1.87, P=.176]$, (please see the Appendix). Thus, there was no difference between positive words associated with their critical lures and negative words associated with their critical lures.

To ensure that the 27 lists of items (351 items in total) we chose had different emotional valences; in a preliminary study we asked other normal subjects to rate those 12 association items in terms of emotional valence. They were instructed to give each item a score of +3 to +1 to represent very positive to slightly positive emotional values, a score of 0 if they perceived no emotional value, and scores of -3 to -1 to represent very negative to slightly negative emotional values, respectively. The results showed that there were significant differences between positive, negative, and neutral item words $[t(49)=28.87, \quad P=.000 ; \quad t(49)=15.71 \quad P=.000 ; \quad t(49)=-20.91$ $P=.000$, respectively]. Table 2 presents the data of comparisons between positive, negative, and neutral lists on the word frequency, association strengths, and emotional valences.

The 27 lists were divided into three sets (sets A, B, and C) for the purpose of counterbalancing. One third of the participants used the $\mathrm{AB}-\mathrm{C}$ form (sets $\mathrm{A}$ and $\mathrm{B}$ were presented to the subjects in the learning phase, and the other set, Set $\mathrm{C}$, was used for unrelated items in the recognition phase); one third of the participants used the BC-A form; and the remaining third of the participants used the CA-B form. Each set included three positive, three negative, and three neutral critical lures. The 27 critical lures (in Chinese) in the experiment and their emotional valences, word frequency, and association strengths are presented in the Appendix. The subjects were asked to rate the items for emotional valence only after they completed the learning and recognition tasks.

\section{LEARNING MATERIALS}

Each list contained 13 items: 12 items (true targets) were presented in order of decreasing associative strength to the nonpresented critical lure item (false target: the 13th item). Eighteen lists of items were presented to the subjects in the learning phase, and the other nine lists (including three positive, three negative, and three neutral lists) were used for unrelated items in the recognition phase. The rationale was that if the subjects had a tendency to answer "old", then the unrelated items would reflect this in the high proportion of "old" responses. ${ }^{[31]}$ Thus, the corrected proportions are equal to the related items minus the unrelated items. ${ }^{[32]}$

\section{RECOGNITION MATERIALS}

The recognition test contained 81 items: 36 studied items or true targets (drawn from the 1st and 7th positions of each of the 18 studied lists) including 12 positive, 12 negative, and 12 neutral items; 18 new unrelated items or true target controls including six positive, six negative, and six neutral items (drawn from the 1st and 7th positions of each of the nine unstudied lists); 18 critical lures or false targets, which were semantically related to the items in each of the 18 studies lists, also including six positive, six negative and six neutral items; and nine new unrelated lures or false target controls (the critical lures for each of the nine unstudied lists).

\section{PROCEDURE}

Subjects were tested individually and were told that they were participating in a study of memory. They were to pay attention to the items that they would see because their memory of these items would be tested later. During the learning phase, each study item was presented visually for $1,700 \mathrm{~ms}$ on the computer screen. A blank screen lasting $1,500 \mathrm{~ms}$ separated each study item from the next. The 18 lists were presented randomly, but followed the order of positive, neutral, and negative with different lists. After the learning phase, the subjects completed simple mathematics problems $10 \mathrm{mi}$ before receiving the recognition test.

For the recognition phase, each of the 81 items was presented visually on the screen for $1,700 \mathrm{~ms}$, with the response options "old" and "new" on the keyboard. After each response, the screen cleared and was followed by a $1,500 \mathrm{~ms}$ delay before the presentation of the next test item.

Finally, we asked the subjects to give those 81 items a score of +3 to +1 to represent very positive to slightly positive emotional values, a score of 0 if they perceived no emotional value, and scores of -3 to

TABLE 2. The comparison between positive, negative, and neutral lists on the word frequency, association strength and emotional valences

\begin{tabular}{lcrrr}
\hline & Positive lists & Negative lists & Neutral lists & $F$-value \\
\hline Word frequency & $89.88(99.24)$ & $83.77(54.84)$ & $69.11(46.67)$ & $0.21(P=.816)$ \\
Association strengths, Mean $(S D)$ & $8.05(1.70)$ & $7.79(1.48)$ & $9.11(1.37)$ & $1.87(P=.176)$ \\
Emotional valences (critical lure only), Mean $(S D)$ & $2.16(0.15)$ & $-2.03(0.29)$ & $0.13(0.13)$ & $950.37(P=.000)$ \\
Emotional valences (12 association items), Mean $(S D)$ & $1.51(0.48)$ & $-1.26(0.37)$ & $0.32(0.23)$ & $122.43(P=.000)$ \\
\hline
\end{tabular}


-1 to represent very negative to slightly negative emotional values, respectively. Subsequently, BDI was administered and they were thanked for their participation.

\section{RESULTS}

\section{TRUE AND FALSE MEMORY}

Table 3 displays the percentage of responses of "old" to the true and false targets in positive, negative, and neutral words, including uncorrected and corrected scores during the recognition procedure.

The raw data (i.e., uncorrected scores of true and false targets, as well as true and false target controls) were analyzed by a $2 \times 3$ (groups $\times$ emotional valence) repeated measures analysis of variance (ANOVA). There was no significant effect between control and depression groups $[F(1,60)=2.26, P=.11$, for true targets; $F(1,60)=0.17, P=.68$, for false targets]. Likewise, neither interaction effect between group and valence was found $[F(2,120)=0.81, P=.45$, for true targets; $F(2,120)=1.70, P=.19$, for false targets]. However, a significant main effect was noted for valence $[F(2,120)=14.49, P<.00$, for true targets; $F(2,120)=5.84, P=.004$, for false targets]. Results of the simple main effect indicated that positive valence items were more significantly recognized than neutral ones for both true and false target scores. The similar analyses were made for true target control and false target control scores. Only a significant effect between valences was observed in false control targets $[F(2,120)=15.27, P<.00]$. The simple main effect indicated more false alarm of positive items $($ Mean $=0.37)$ than of negative ones, and more false alarm of negative items (Mean $=0.24)$ than of neutral ones $($ Mean $=0.12)$.

Meanwhile, we also analyzed corrected data of true and false target scores as shown in the conventional literature of the DRM paradigm task. The purpose of the corrective procedure was to eliminate differences in the subjects' criteria in judging whether an item was "old" or "new"; we used unrelated items as the index to correct the proportions. ${ }^{[31,32]}$ As previously mentioned, the corrected scores are equal to the related items minus the unrelated items, that is, the corrected true recognition scores are equal to the true target scores minus the true target control scores (unrelated items), and corrected false recognition scores are equal to the false target scores minus the false target control scores (unrelated critical lures).

The data were then analyzed for depressive disorder and control subjects by a $2 \times 3$ (groups $\times$ emotional valence) repeated measures ANOVA. As for the true recognition scores, no interaction between group and valence was found $[F(2,120)=1.08, P=.52]$. No main effect between the depression and normal group was found $[F(1,60)=2.52, P=.12]$. A significant main effect was found for valence $[F(2,120)=3.22, P=.03]$. Results of the simple main effect indicated that positive valence items were recognized more significantly than neutral ones in the normal group $[F(2,58)=3.41$, $P=.04]$. No simple main effect in the depression group was found $[F(2,62)=1.65, P=.20]$.

As for the false recognition scores, no interaction among group and valence was found $[F(2,120)=1.22$, $P=.65]$. A significant main effect was found for a group $[F(1,60)=4.12, P=.02]$, in that the depression group recognized more false targets than the control group. A significant main effect was also found for valence $[F(2,120)=4.96, P=.01]$. Results of the simple main effect indicated that positive and negative valence items were recognized significantly less than neutral ones in the normal group $[F(2,58)=5.22, P=.01)$. No simple main effect in the depression group was found $[F(2,62)=1.02$, $P=.41]$. When compared to the normal group, the depression group showed a greater tendency to judge the emotional (positive and negative) items as "old" than neutral items. That is, subjects with depressive disorders more often incorrectly claimed that they had seen the emotional items than the control group did.

\section{MEMORY DISCRIMINATION AND RESPONSE CRITERION}

To compare memory performance in the depression and control groups, the data were calculated,

TABLE 3. Percentage of items judged "old" in the true and false recognition of the depression and control groups

\begin{tabular}{|c|c|c|c|c|c|c|}
\hline & \multicolumn{3}{|c|}{ Uncorrected } & \multicolumn{3}{|c|}{ Corrected } \\
\hline & True target & False target & True target control & False target control & True target & False target \\
\hline \multicolumn{7}{|l|}{ Depression } \\
\hline Positive & 0.68 & 0.54 & 0.28 & 0.30 & 0.40 & 0.23 \\
\hline Negative & 0.68 & 0.49 & 0.28 & 0.24 & 0.40 & 0.25 \\
\hline Neutral & 0.54 & 0.42 & 0.24 & 0.09 & 0.30 & 0.33 \\
\hline \multicolumn{7}{|l|}{ Control } \\
\hline Positive & 0.72 & 0.56 & 0.23 & 0.44 & 0.50 & 0.12 \\
\hline Negative & 0.77 & 0.38 & 0.31 & 0.24 & 0.45 & 0.15 \\
\hline Neutral & 0.65 & 0.43 & 0.24 & 0.15 & 0.41 & 0.28 \\
\hline
\end{tabular}

True target, association items in each studied list; False target, the nonpresented critical lure. True target control (unrelated items), association items in each unstudied list; False target control (unrelated lures), the nonpresented critical lure in each unstudied list. 
TABLE 4. Memory discrimination index $\left(d^{\prime}\right)$ and response criterion $(C)$ in the depression and control groups for true target

\begin{tabular}{lcc}
\hline & Discrimination index $\left(d^{\prime}\right)$ & Response criterion $(C)$ \\
\hline Depression & & \\
$\quad$ Positive & 1.51 & 0.44 \\
Negative & 1.38 & 0.18 \\
$\quad$ Neutral & 1.56 & 0.31 \\
Control & & \\
Positive & 2.21 & 0.13 \\
Negative & 1.81 & 0.25 \\
Neutral & 1.79 & 0.34 \\
\hline
\end{tabular}

1. $d^{\prime}$ and $C$ are measures of accuracy and bias from signal detection theory. ${ }^{[27]}$

2. A higher $d^{\prime}$ score indicates a greater ability to discriminate.

3 . For the $C$ score, a zero value indicates a neutral criterion; a positive value indicates a conservative criterion; and a negative value indicates a liberal criterion.

$d^{\prime}$, a measure of memory discrimination (for false target, $d^{\prime}$ indicates false recognition susceptibility), and $C$, a measure of response criterion. ${ }^{[27]}$ The values of $d^{\prime}$ and $C$ across the depression and control groups are displayed in Table 4. A higher $d^{\prime}$ score indicates a greater ability to discriminate on the part of the subjects. For the $C$ score, a zero value indicates a neutral criterion; a positive value indicates a conservative criterion; and a negative value indicates a liberal criterion. ${ }^{[27]}$

The data were then analyzed for depressive disorder and control subjects by a $2 \times 3$ (groups $\times$ emotional valences) repeated measures ANOVA. Table 4 displays the result for true targets scores. The memory discrimination $\left(d^{\prime}\right)$ score served as the dependent variable. There is a significant difference between groups $[F(1,60)=4.36, P=.02]$, indicating that the depression group had the less memory discrimination than the control group. No other significant was found $[F(2,120)=0.38, \quad P=.69$, for interaction between group and emotional valence; $F(2,120)=0.49$, $P=.62$, for emotional valence]. The same analysis was done for $\mathrm{C}$ scores. An interaction effect among groups and valence was found $[F(2,120)=4.72$, $P=.01]$. Results of the simple main effect indicated that when compared with normal subjects $($ Mean $=0.13)$, depression patients $($ Mean $=0.44)$ judged the positive items with more conservative criterion $[t(1,60)=3.18, P=.01]$. No significant was found in the negative items lists between groups $[t(1$, $60)=1.81, P=.11]$.

\section{EMOTIONAL INTENSITY}

The emotional intensity scores of the 81 recognition items across the depression and control groups are displayed in Table 5 . The data were subjected to a 2
TABLE 5. Emotional valences of 81 recognition items in the depression and control groups

\begin{tabular}{lrr}
\hline & True target & False target \\
\hline Depression & & \\
Positive & 1.00 & 1.13 \\
Negative & -1.02 & -1.60 \\
Neutral & 0.42 & -0.18 \\
Control & & \\
Positive & 1.63 & 1.94 \\
Negative & -1.26 & -2.05 \\
Neutral & 0.20 & 0.13 \\
\hline
\end{tabular}

(Group) $\times 3$ (Emotional valence) way mixed ANOVA. The emotional intensity score served as the dependent variable.

For true targets, an interaction effect among groups and valence was found $[F(2,120)=3.85, P=.03]$. Results of the simple main effect indicated that emotional valences were significantly different in both groups $[F(2,60)=112.98, P=.000$, for the normal group; $F(2,60=20.00, P=.000$, for the depression group]. When compared with the normal group $($ Mean $=1.63)$, the depression group perceived the positive items as having less positive intensity $($ Mean $=1.00)[t(1,60)=2.67, P=.01]$.

For false targets, an interaction effect among groups and valence was also found $[F(2,120)=5.70, P=.005]$. Results of the simple main effect indicated that emotional valences were significantly different in both groups $[F(2,60=162.59, P=.000$, for normal group; $F(2,60=28.36, P=.000$, for depression group]. Once again, when compared with normal group $($ Mean $=1.94)$, the depression group perceived positive items with less positive emotional intensity $($ Mean $=1.13)[t(1,60)=3.14, P=.003]$.

\section{DISCUSSION}

On the basis of conventional data analyses for the DRM paradigm task (i.e., using corrected false memory scores for data analyses), the results revealed that our subjects with depression disorder had more negative and positive false memories than their healthy controls. This study thus partially supports the first hypothesis; subjects with depression disorder had more false memories than healthy controls. This was not a result of poor veridical memory performances of depressed patients, for our data showed that there was no difference in true target scores between the depressed and normal groups. The second aim was to examine whether depressed patients indeed activate negative association memories, as MCM suggests. The results showed that there was no interaction effect among groups and emotional valence. There were no response differences between patients with depression disorders 
and healthy controls among positive, negative, and neutral lists. Thus, we failed to demonstrate the presence of the MCM bias in the false memory paradigm.

We found that depressed subjects had more false memories than control subjects; they often incorrectly claimed that they had seen the critical lures regardless of the emotional valences of the list materials. This partially supports that the cognitive styles of overgeneralization and rumination in depression could make negative false memory more accessible than veridical memory.

There are some theories that attempt to explain false memory. Underwood ${ }^{[33]}$ explained such a robust phenomenon with the implicit associative response; that is, when subjects encode serial lists of associated words in the learning phase, they think of semantic connections to those words. In the recognition phase, the subjects activate representations for related critical lures (false targets) because those words are the highest semantic associates of the list items. ${ }^{[33,34]}$ The source monitoring framework focuses on the processes by which people differentiate between memories of actual and imagined experiences. ${ }^{[35]}$ According to the source monitoring framework, false memory phenomena arise when thoughts, images, and feelings from one source are attributed to another erroneous source. Another theory is known as the fuzzy trace theory, ${ }^{[36,37]}$ which maintains that people develop two kinds of memory "trace": verbatim traces, which record surface-level sensory aspects of experience, and gist traces, which record more abstract or general glosses of input, semantic meanings, interpretations, and elaborations of experience. False recognitions are explained as follows: at recall, subjects activate the general gist of their memory for the list. ${ }^{[37]}$ Our results show more false memory in subjects with depressive disorders than in normal subjects. According to these theories, associated items may have activated more false conceptual targets (or gist traces) and reduced the monitoring ability of subjects with depressive disorders.

It is suggested that overgeneralization and rumination cognitive styles partially play a role in the activation of abstract semantic meanings and false interpretations in the recognition stage. This familiarity or gist-based recognition style may distort what depressed patients remember and generate incorrect inferences about their current and future situations. ${ }^{[38]}$

MCM bias was proposed as a possibly important maintenance mechanism in depression. ${ }^{[8]}$ However, we did not find this form of memory bias in the clinically depressed subjects, neither in the true nor in the false recognition responses. Indeed, studies on MCM bias with different tasks have produced mixed findings. In early discussions, ${ }^{[39]}$ explained that depression is characterized by a negative bias in elaborative processes in memory, which require effortful and attention control (e.g., explicit memory tasks), but not in activation processes, which are automatic and outside awareness (e.g., implicit memory tasks). Results that fail to support MCM bias in implicit memory have been found in recent studies. ${ }^{[1,40-45]}$ argued that it might be due to the different perceptual and conceptual processing in the encoding stage of implicit memory. It is possible that the material items used in this study were context free (two words), which could not fully activate mood-congruent effects. Procedures providing context, such as autobiographical memories involving self-referent information may be more useful in this regard. ${ }^{[39,46,47]}$

It was noted that the control group had more scores of false target control (the lures of unrelated lists) in the positive lists (0.44) than in the depression group (Table 3). This reduces the corrected scores of false target (0.12) in the normal group. As the unrelated lists were not presented to the subjects, it could not activate the positive critical lures. Thus, the scores did not reflect the conventional definition of false memory in the DRM paradigm. Normal subjects had a tendency to say "yes" to positive unrelated lures, although it does not have the semantic meanings to the previous memory items. Similar results came from some studies, which found that normal subjects recall more positive items than negative items. ${ }^{[46,48]}$ explained the reason why normal people always claim that they are happy. However, it needs more research to explore this issue.

We did not find any memory discrimination differences between the normal and depression group. However, the results of this study indicate a difference in judgment criteria of emotional lists between the depressed and control group, as Table 4 shows. When compared with the normal group, the depression group had more conservative criteria in the positive lists. It was found that depression subjects had more conservative criteria when they judged the positive true targets (i.e., I don't think that I have seen this positive item before even though it is familiar to me), whereas the normal subjects did not show this judgment pattern. The difference criteria suggest that the depressed subjects distorted the positive information when making a judgment; meanwhile, they treated positive information with stricter criterion than normal subjects.

When compared with the normal group, the depression group perceived positive items as having less positive intensity. It seems that the positive information did not reflect "a truly positive item" for subjects with depressive disorders. This phenomenon could be explained by the tripartite model of depression, ${ }^{[49]}$ which suggests that a "low positive effect" is characterized by a cluster of symptoms relatively specific to depression, such as anhedonia, lack of energy, and disinterest. Thus, memories about positive information no longer have positive connotations for depressed individuals. The MCM bias supposes that emotional memory retrieval is affected by a subject's mood; 
however, the present results suggest that emotional memory intensity is attenuated by a subject's mood. ${ }^{[46,48]}$ proposed the difference in emotional intensity between normal and depressed subjects with the "faded affect bias". They investigated positive and

\section{Appendix}

The 27 critical lures (in Chinese) in the experiment and their emotion valences, word frequency, and association strengths (see Table A1).

TABLE A1.

\begin{tabular}{|c|c|c|c|c|c|c|c|c|c|}
\hline \multirow[b]{2}{*}{ Positive } & \multicolumn{3}{|c|}{ Set A } & \multicolumn{3}{|c|}{ Set B } & \multicolumn{3}{|c|}{ Set $\mathrm{C}$} \\
\hline & Art & Reward & Satiation & Passion & Respect & Braveness & Welfare & Romantic & Tenderness \\
\hline Word frequency & 347 & 32 & 58 & 75 & 104 & 39 & 73 & 49 & 32 \\
\hline Association strengths & 7.25 & 8.25 & 7.5 & 6.75 & 6.33 & 7.08 & 12.0 & 8.33 & 9.00 \\
\hline Emotion valences (critical lure only) & 2 & 2.16 & 2.06 & 2.21 & 2.11 & 2.11 & 2.44 & 2.0 & 2.35 \\
\hline Emotional valences (12 association items) & 1.87 & 2.19 & 1.25 & 0.91 & 1.58 & 0.70 & 1.91 & 1.67 & 1.54 \\
\hline Negative & Typhoon & Censure & Sufferance & Violence & Pollution & Annoyance & Suicide & Destruction & Deterioration \\
\hline Word frequency & 35 & 32 & 200 & 99 & 80 & 33 & 112 & 112 & 51 \\
\hline Association strengths & 9.33 & 7.08 & 6.58 & 6.5 & 10 & 7 & 7 & 6.75 & 9.92 \\
\hline Emotion valences (critical lure only) & -1.58 & -1.78 & -2.06 & -1.94 & -2.15 & -2.09 & -2.61 & -1.91 & -2.19 \\
\hline Emotional valences (12 association items) & -1.20 & -1.25 & -1.16 & -1.83 & -0.75 & -1.37 & -1.83 & -0.87 & -1.08 \\
\hline Neutral & Glasses & Tourist & Spread & Road & Category & Business owner & Revolve & Cost & Railway \\
\hline Word frequency & & 52 & 67 & 46 & 46 & 99 & 39 & 183 & 53 \\
\hline Association strengths & 6.75 & 8.83 & 9.17 & 10.33 & 8.58 & 8.25 & 11.08 & 8.33 & 10.67 \\
\hline Emotion valences (critical lure only) & 0.03 & 0.06 & 0.4 & 0.09 & 0 & 0.2 & 0.06 & 0.03 & 0.28 \\
\hline Emotional valences (12 association items) & 0.08 & 0.41 & 0.37 & -0.15 & 0.45 & 0.62 & 0.42 & 0.45 & 0.25 \\
\hline
\end{tabular}

negative emotional memory in depressed subjects who recalled six emotionally intense memories from their lives and provided a series of ratings for each event; as time passed, the unpleasant emotions faded more than the pleasant emotions in the normal group. However, the unpleasant emotional memory did not fade in depressed subjects.

In conclusion, based on the corrected scores in DRM paradigm, we found more false memories in subjects with depressive disorders compared with healthy controls while we failed to demonstrate more negative false recognition in depression. The depression group judged the positive items with more conservative criteria. We also found that positive information did not reflect "the truly positive item" for subjects with depressive disorders. Thus, it is suggested that emotional memory valences are attenuated by patients' mood.

Acknowledgments. This research was partly supported by Grants NSC92-2413-H-002-011 and NSC93-2413-H-002-013 from the National Science Council, Taipei, Taiwan, as well as by Cognitive Neuroscience Development Grant from College of Science, National Taiwan University, Taipei, Taiwan.

\section{REFERENCES}

1. Banos R, Medina P, Pascual J. Explicit and implicit memory biases in depression and panic disorder. Behav Res Ther 2001;39:61-74.

2. Calev A. Affect and memory in depression: evidence of better delayed recall of positive than negative affect words. Psychopathology 1996;29:71-76.

3. Murray LA, Whitehouse WG, Alloy LB. Mood congruence and depressive deficits in memory: a forced-recall analysis. Memory 1999;7:175-196.

4. Watkins E, Brown R. Rumination and executive function in depression: an experimental study. J Neurol Neurosurg Psychiatr 2002;72:400-402.

5. Blaney PH. Affect and memory: a review. Psychol Bull 1986;99:229-246.

6. Eich E, Macaulay D. Are real moods required to reveal moodcongruent and mood-dependent memory? Psychol Sci 1991;11:244-248.

7. Rothkopf JS, Blaney PH. Mood congruent memory: the role of affective focus and gender. Cogn Emotion 1991;5:53-64.

8. Teasdale JD. Negative thinking in depression: cause, effect or reciprocal relationship? Adv Behav Res Ther 1983;5: $3-25$.

9. Edelman RE, Ahrens AH, Haaga DA. Inferences about the self, attributions, and overgeneralization as predictors of recovery from dysphoria. Cogn Ther Res 1994;18:551-566. 
10. Ganellen RJ. Specificity of attributions and overgeneralization in depression and anxiety. J Abnorm Psychol 1988;97: 83-86.

11. Lyubomirsky S, Kasri F, Zehm K. Dysphoric rumination impairs concentration on academic tasks. Cogn Ther Res 2003;27: 309-330.

12. Nolen-Hoeksema S. The role of rumination in depressive disorders and mixed anxiety/depressive symptoms. J Abnorm Psychol 2000;109:504-511.

13. Robinson MS, Alloy LB. Negative cognitive styles and stressreactive rumination interact to predict depression: a prospective study. Cogn Ther Res 2003;27:275-292.

14. Wenzlaff RM, Luxton DD. The role of thought suppression in depressive rumination. Cognit Ther Res 2003;27:293-308.

15. Beck AT. Cognitive Therapy and the emotional disorders. New York: International Universities Press; 1976.

16. Nolen-Hoeksema S. Responses to depression and their effects on the duration of depressive episodes. J Abnorm Psychol 1991;100: $569-582$.

17. Watkins E, Teasdale JD. Rumination and overgeneration in depression: effects of self-focus and analytic thinking. J Abnorm Psychol 2001;110:353-357.

18. Schacter DL. False recognition and the brain. Curr Dir Psychol Sci 1997;6:65-70.

19. Moritz S, Woodward TS, Cuttler C, Whitman JC, Watson JM. False memories in schizophrenia. Neuropsychology 2004;18: 269-725.

20. Schacter DL, Verfaellie M, Anes MD. Illusory memories in amnesic patients: conceptual and perceptual false recognition. Neuropsychology 1997;11:331-342.

21. Balota DA, Cortese MJ, Duchek JM, Adams D, Roediger HL, McDermott KB. Veridical and false memories in healthy older adults and in dementia of the Alzheimer's type. Cogn Neuropsychol 1999;16:361-384.

22. Budson AE, Todman RW, Chong H, Adams EH, Kensinger EA, Krangel TS, Wright CI. False recognition of emotional word lists in aging and Alzheimer disease. Cogn Behav Neurol. 2006;19:71-78.

23. Pesta BJ, Murphy MD, Sanders RE. Are emotionally charged lures immune to false memory? J Exp Psychol Learn Mem Cogn 2001;27:328-338.

24. Kensinger EA, Corkin S. The effects of emotional content and aging on false memories. Cogn Affect Behav Neurosci 2004:4:1-9.

25. Deese J. On the prediction of occurrence of particular verbal intrusions in immediate recall. J Exp Psychol 1959;58: $17-22$.

26. Roediger HL, McDermott KB. Creating false memories: remembering words not presented in lists. J Exp Psychol Learn Mem Cogn 1995;21:803-814.

27. Snodgrass JG, Corwin J. Pragmatics of measuring recognition memory: applications to dementia and amnesia. J Exp Psychol Gen 1988;117:34-50.

28. Sheehan DV, Lecrubier Y, Sheehan K, Amorim P, Janavs J, Weiller E. The Mini-International Neuropsychiatric Interview (M.I.N.I): the development and validation of a structured diagnostic psychiatric interview for DSM-IV and ICD-10. J Clin Psychiatry 1998;59:22-33.

29. Chen HC. The Chinese words association norm (Annual research report of designated subject). Taipei, Taiwan: National Science Council of Taiwan; 1999.
30. Li H, Li TK, Tseng JF. The Chinese dictionary words frequency statistic. Taipei, Taiwan: Ministry of Education in Taiwan; 1997.

31. Schacter DL, Cendan DL, Dodson CS, Clifford ER. Retrieval conditions and false recognition: testing the distinctiveness heuristic. Psychon Bull Rev 2001;8:827-833.

32. Budson AE, Sitarski J, Daffner KR, Schacter DL. False recognition of pictures versus words in Alzheimer's disease: the distinctiveness heuristic. Neuropsychology 2002;16:163-173.

33. Underwood BJ. False recognition produced by implicit verbal responses. J Exp Psychol 1965;70:122-129.

34. Hancock TW, Hicks JL, Marsh RL, Ritschel L. Measuring the activation level of critical lures in the Deese-Roediger-McDermott paradigm. Am J Psychol 2003;116:1-14.

35. Lindsay DS, Johnson MK. False memories and the source monitoring framework: reply to Reyna and Lloyd. Learn Individ Differences 2000;12:145-161.

36. Brainerd C, Reyna V, Brandse E. Are children's false memories more persistent than their true memories? Psychol Sci $1995 ; 6: 359-364$.

37. Reyna VF. Fuzzy-trace theory and source monitoring: an evaluation of theory and false-memory data. Learn Individ Differences 2000;12:163-175.

38. Moritz S, Glascher J, Brassen S. Investigation of moodcongruent false and true memory recognition in depression. Depress Anxiety 2005;21:9-17.

39. Williams J, Scott J. Autobiographical memory in depression. Psychol Med 1988;18:689-695.

40. Danion JM, Kauffmann-Muller F, Grange D, Zimmerman MA, Greth P. Affective valence of words, explicit and implicit memory in clinical depression. J Affect Disord 1995;34:227-234.

41. Dunn BD, Dalgleish T, Lawrence AD, Cusack R, Ogilvie AD. Categorical and dimensional reports of experienced affect to emotion-inducing pictures in depression. J Abnorm Psychol 2004;113:654-660.

42. Tarsia M, Power MJ, Sanavio E. Implicit and explicit memory bias in mixed anxiety-depression. J Affect Disord 2003;77:213-225.

43. Watkins P C, Mathews A, Williamson DA, Fuller RD. Moodcongruent memory in depression: emotional priming or elaboration? J Abnorm Psychol 1992;101:581-586.

44. Watkins PC, Martin CK, Stern LD. Unconscious memory bias in depression: perceptual and conceptual processes. J Abnorm Psychol 2000;109:282-289.

45. Watkins PC. Implicit memory bias in depression. Cogn Emotion 2002;16:381-402.

46. Walker WR, Skowronski JJ, Gibbons JA, Vogl RJ, Thompson CP. On the emotions that accompany autobiographical memories: dysphoria disrupts the fading affect bias. Cogn Emotion 2003;17:703-723.

47. Williams J, Broadbent K. Autobiographical memory in suicide attempters. J Abnorm Psychol 1986;95:144-149.

48. Walker WR, Skowronski JJ, Thompson CP. Life is pleasantand memory helps to keep it that way. Rev Gen Psychol 2003;7:203-210.

49. Clark LA, Watson D. Tripartite model of anxiety and depression: psychometric evidence and taxonomic implications. J Abnorm Psychol 1991;100:316-336.

50. Norman KA, Schacter DL. False recognition in younger and older adults: exploring the characteristics of illusory memories. Mem Cognit 1997;25:838-848. 